\title{
Probabilistic strength assessment through analytical modelling of pillars found in ship structures
}

\author{
Angelos S. Vasileiou ${ }^{1}$ Konstantinos N. Anyfantis ${ }^{1, *}$ \\ ${ }^{1}$ Shipbuilding Technology Laboratory, School of Naval Architecture and Marine Engineering, \\ National Technical University of Athens, 106 82, Athens, Greece
}

\begin{abstract}
The compressive strength of pillars found in ship structures is studied under a reliability perspective. Monte Carlo Simulations (MCS) are applied, aided by a stratified sample scheme (i.e. Latin Hypercube), to account for uncertainty within the problem's input variables (yield stress, elastic modulus, initial bow imperfection). MCSs were applied for three slenderness ratio values (low, medium, high), for hollow-circular, hollowsquare and " $\mathrm{H}$ " shape cross-sections, and multiple geometries per slenderness ratio, per cross-section. The pillar's strength is calculated based on the Perry-Robertson formula. The probabilistic resistance per pillar was modelled by generating the probability density function that best describes the statistical nature of the sample data. In this paper we illustrate the probabilistic nature of the compression column resistance, and compare it to the deterministic resistance suggested by regulatory bodies.
\end{abstract}

\section{Introduction}

In shipbuilding, pillars are typically used to support large deck areas in car carriers, passenger ships and general cargo vessels as well as in the accommodation area in merchant vessels. Reliable design against their ultimate compressive capacity is therefore of high interest. Their typical scantlings are characterized by intermediate slenderness ratios that lead to elastoplastic buckling, which is a collapse mode that combines material and geometric nonlinearities [1]. Rule-based (e.g. Common Structural Rules-CSR in [2]) strength check involves the application of the well-established Euler-Johnson buckling formula with a maximum allowable utilization factor of 0.65 for static loads and 0.75 for dynamic loads. The utilization factor deterministically accounts for the uncertainties involved within the computation of a pillar's nominal capacity. Therefore, the employment of the $65 \%$ (static) and $75 \%$ (dynamic) compressive strength is allowed to be considered during the design phase. This design practice ascertains that the dimensioned pillars will succeed to fulfil their design purpose during their service life. Nevertheless, pillar failure may occur without though jeopardizing the global and strength stability of vessel. It is of interest therefore to assess a pillar's strength from the probabilistic point of view and consequently manage to assign a

* Corresponding author: kanyf@naval.ntua.gr 
probability of failure to a given design. This design perspective requires the application of notions associated within the field of structural reliability [3].

In shipbuilding, dimensional tolerances placed on the scantlings of a pillar's cross section are quite tight [4] and therefore corresponding dimension uncertainty may be neglected. On the other hand, there does exist a significant variability in material related parameters ([5 - 6]) and out-of-straightness initial deflections [7 - 9], which have a direct impact on the pillar's compressive strength. This study has placed effort on propagating the variability in the material's Young's modulus and yield strength along with the variability found in the initial geometric imperfection to the pillar's capacity. Based on a probabilistic framework, the statistical structure of pillars, generalized with respect to their slenderness ratios, is constructed following a series of Monte Carlo Simulations (MCS). Finally, the probability of failure, based on the deterministic rule-based compression strength (CSR) with a maximum utilization factor of 0.65 , was estimated based on the derived statistical structure.

\section{Theoretical background}

In order to able to provide deterministic strength calculations of pillars, within the MCS, the analytical solution derived by Perry and Robertson [1] is considered within this work.

\subsection{Deterministic calculation of compressive strength}

A pillar is a compression member with prismatic geometry that under idealized conditions is assumed to be straight and free of residual stresses. In such a foundation, Euler's buckling theory provides the critical elastic compressive load. In reality pillars are not perfectly straight, due to their manufacturing, transportation and handling processes. Typical realistic out-of-straightness shapes are illustrated in Figure 1, in a quite magnified scale. The exact pillar's geometric imperfection is dependent on its length, cross-section geometry and its production (forging, rolling, welding etc.). In order to simplify the structural analysis of a pillar by incorporating its initial deflection within the problem's formulation, the real complicated deflections are converted to one, sinusoidal single wave along the column's main axis as shown in Figure 1. This assumption is performed on the basis that such analytical description is equivalent to the theoretical critical eigenmode calculated from an elastic buckling analysis.
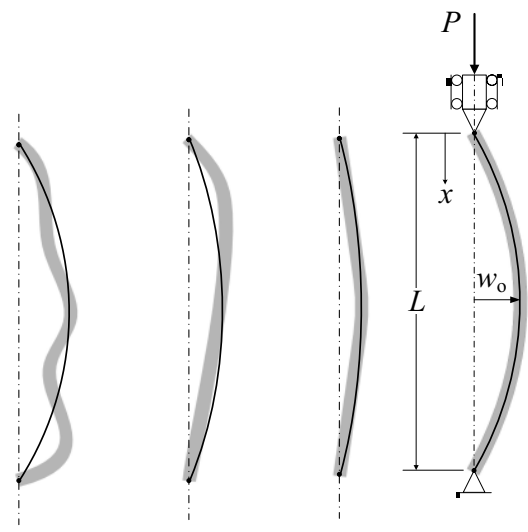

Fig. 1 Measured initial out-of-straightness deflection of a pillar (three left to right schematics) and corresponding assumed field for establishing structural analsysis (most right schematic). 
The initial considered deflection along the pillar's length $L$ is described by:

$$
w_{\mathrm{o}}(x)=w_{\mathrm{o}} \sin \left(\frac{\pi x}{L}\right)
$$

where the maximum deflection, $w_{0}$, is located at mid-span. The equation of the column's elastic deflection curve is written as:

$$
\frac{\mathrm{d}^{2} w(x)}{\mathrm{d} x^{2}}=\frac{M}{E I}=-\frac{P}{E I}\left(w(x)+w_{\mathrm{o}}\right)
$$

Where $P$ is the external compressive load, $E$ is the Young's modulus and $I$ is the crosssectional moment of inertia about the axis normal to the buckling plane. The general solution of the above differential equation gives a description of the total deflection at mid-span:

$$
w(x)=A \sin (p x)+B \cos (p x)+\frac{p^{2} w_{\mathrm{o}}}{\left(\frac{\pi}{L}\right)^{2}-\left(\frac{P}{E I}\right)^{2}} \sin \left(\frac{\pi x}{L}\right)
$$

In the case of simply supported edges, the boundary condition describing the problem are $w$ $(0)=0$ and $w(L)=0$. Therefore, Eq.(3) becomes:

$$
w(x)=\frac{w_{\mathrm{o}}}{P_{\mathrm{E}} / P-1} \sin \left(\frac{\pi x}{L}\right)
$$

Where $P_{\mathrm{E}}$ is the elastic buckling critical load (Euler load), equal to $\pi^{2} E I / L^{2}$. The maximum initial deflection is magnified at mid-span following the pillar's loading and Eq. (4) may be rewritten as:

$$
w_{\max }=w(x=L / 2)=\frac{w_{\mathrm{o}}}{P_{\mathrm{E}} / P-1}
$$

The preceding equation does not provide any additional information on the maximum compressive load, apart from that already given by the Euler load. However, having knowledge on the actual non-linear lateral deflection, one can easily associate a corresponding stress field. Two main stress components are superimposed over the depth of the pillar' cross-section (or along the direction normal to weakest axis). An average uniform compressive field resulting from the axial load and a linear varying one from the secondary moment (see Fig. 2) coexist.

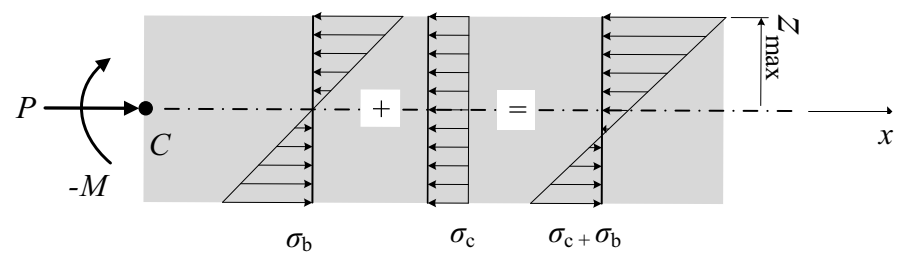

Fig. 2 Induced cross-section moment and stress field due to the imposed compressive load $\mathrm{P}$ at a pillar with initial deflection 
In this respect, Perry-Robertson formula provides a handy relation that can be used for the evaluation of the critical average compressive stress, $\sigma_{\mathrm{cr}}$, as:

$$
\sigma_{\mathrm{cr}}=\frac{1}{2}\left[\sigma_{\mathrm{y}}+(1+\eta) \sigma_{\mathrm{E}}\right]-\sqrt{\frac{1}{4}\left[\sigma_{\mathrm{y}}+(1+\eta) \sigma_{\mathrm{E}}\right]^{2}-\sigma_{\mathrm{y}} \sigma_{\mathrm{E}}}
$$

where $\sigma_{\mathrm{y}}$ is the material yield strength, $\sigma_{\mathrm{E}}$ is the Euler stress $\left(P_{\mathrm{E}} / A, A\right.$ being the section's cross sectional area) and $\eta$ the cross-section imperfection coefficient, which is given by:

$$
\eta=\frac{w_{\mathrm{o}} z_{\max }}{r^{2}}
$$

where $z_{\max }$ is the distance of the most remote material fiber from the section's neutral axis and $r$ is the radius of gyration. Perry's formula associates the maximum attained stress within the pillar's 1D analysis with the applied compressive load that causes this stress to arise. Hence magnitude $\sigma_{\mathrm{cr}}$ corresponds at the applied stress level that corresponds at the first yield of the pillar. This stress level is quite close to the actual collapse load [1].

On the other hand, CSR prescriptive calculations for a pillar's compressive strength [2] employ the unified Euler-Johnson buckling criterion (Eq. 8) with a maximum allowable utilization factor, $\eta_{\text {all. }}$, for static loading equal to 0.65 . It is noted that this formula do not explicitly account for the actual initial imperfection magnitude.

$$
\left\{\begin{array}{lll}
\sigma_{\mathrm{cr}}=\sigma_{\mathrm{E}} & \text { if } & \sigma_{\mathrm{E}} \leq \sigma_{\mathrm{y}} / 2 \\
\sigma_{\mathrm{cr}}=\sigma_{\mathrm{y}}\left(1-\sigma_{\mathrm{y}} / 4 \sigma_{\mathrm{E}}\right) & \text { if } & \sigma_{\mathrm{E}} \leq \sigma_{\mathrm{y}} / 2
\end{array}\right\}
$$

\section{Uncertainty definition and propagation}

A pillar's compressive strength or resistance, within the terminology of structural reliability, depends on geometrical and material parameters which in reality are not deterministic quantities but differ from part to part as a result of production and assembly related processes. Within a structural reliability framework these may be considered as uncertainties that obey a given statistical structure. In this direction, the Young's modulus, $E$, the yield stress, $\sigma_{\mathrm{y}}$, and the initial bow imperfection, $w_{\mathrm{o}}$, were considered as Random Variables (RVs). Therefore, the pillar's resistance may be expressed in probabilistic terms through the uncertainty propagation of the input parameters. As it will be shown in the last section, a probability of failure, $p_{\mathrm{f}}$, may be linked to a given resistance range (i.e. occurrence in probability theory).

\subsection{Uncertainty in initial imperfection}

According to the International Association of Classification Societies (IACS), typical values of the maximum initial bow imperfection of support members between ship decks is allowed to be maximum $4 \mathrm{~mm}$ at standard operating conditions, with $6 \mathrm{~mm}$ being the upper allowable limit [4]. Also according to experimental studies, the initial column deflection at the column mid-span follows the lognormal distribution [7], having the following functional form:

$$
f\left(w_{\mathrm{o}}\right)=\left\{\begin{array}{l}
\frac{1}{\sqrt{2 \pi} \sigma w_{\mathrm{o}}} \exp \left[-\frac{1}{2}\left(\frac{\ln w_{\mathrm{o}}-\mu}{\sigma}\right)^{2}\right] \\
0 \\
w_{\mathrm{o}}>0 \\
\text { elsewhere }
\end{array}\right\}
$$


Where the mean value $m$ and variance $v$ are a function of the parameters $\mu$ and $\sigma$ as follows:

$$
\begin{aligned}
& m=\exp \left(\mu+\frac{\sigma^{2}}{2}\right) \\
& v=\exp \left(2 \mu+\sigma^{2}\right)\left[\exp \left(\sigma^{2}\right)-1\right]
\end{aligned}
$$

For the estimation of a pillar's resistance, without loss of generality and according to the above, the shape of the distribution derives from the following assumptions: The initial column maximum deflection may be less than $4 \mathrm{~mm}$ in $\sim 95 \%$ of the observations, with $\sim 99 \%$ of the columns having an initial deflection of less than $6 \mathrm{~mm}$. The values of the parameters can be retrieved from the following system of equations.

$$
\left\{\begin{array}{l}
F\left(w_{\mathrm{o}}=4\right)=\Phi\left(\frac{\ln 4-\mu}{\sigma}\right)=0.95 \\
F\left(w_{\mathrm{o}}=6\right)=\Phi\left(\frac{\ln 6-\mu}{\sigma}\right)=0.99
\end{array}\right\}
$$

Where $\Phi$ is the cumulative distribution function of the standard normal distribution (i.e. $N(0,1))$. Therefore, the initial column maximum deflection shall follow the lognormal distribution as given in Fig. 3.

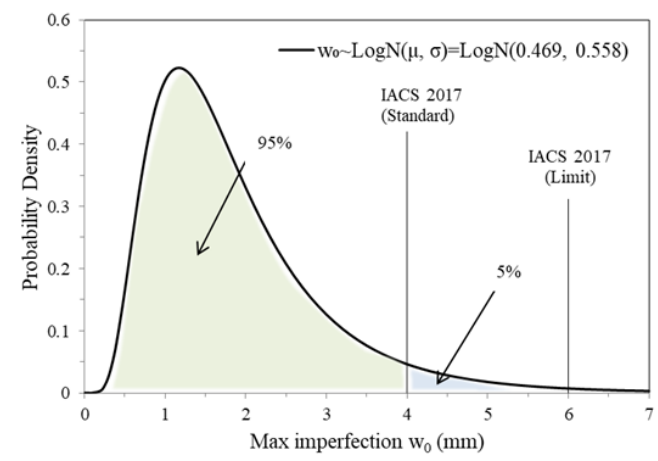

Fig. 3 Initial bow imperfection considered statistical structure.

\subsection{Uncertainty in material properties}

According to experimental results for steel grade A (nominal yield stress equal to $235 \mathrm{MPa}$ ), which is commonly used in pillars found in ship structures, the yield strength may follow the normal or lognormal distribution, with a mean value of $284.5 \mathrm{MPa}$ and a standard deviation of $21.5 \mathrm{MPa}$ [6]. To account for the fact that yield stress takes only positive values, the lognormal distribution was used instead, which follows the formula of Eq. (13). Given the mean value $m$ and standard deviation $v$, the lognormal distribution parameters $\mu$ and $\sigma$ can be calculated through statistics. The probability density function representing the yield strength distribution has therefore the following parameters:

$$
\sigma_{\mathrm{y}} \sim \operatorname{Lognormal}\left(\mu=5.648, \sigma^{2}=0.076^{2}\right)
$$


According to experimental results, the Young's elastic modulus of steel grade S235 may follow the normal distribution, with a mean value of $210 \mathrm{GPa}$ and a standard deviation of $12.6 G P a[2]$. The probability density function of the elastic modulus is given by:

$$
E \sim \operatorname{Normal}\left(\mu=210, \sigma^{2}=12.6^{2}\right)
$$

\subsection{Uncertainty propagation and sampling schemes}

Uncertainty propagation was established through a Monte Carlo Simulation (MCS) assisted by the employment of a stratified sampling scheme, i.e. Latin Hypercube. For illustration purposes, Figure 4 presents joint pair distributions of the input RVs for a sampling size of $10^{4}$ observations, according to which convergence was achieved (see Figure 5). The resistance was calculated per observation in a deterministic manner by employing Eq. 6, which is a function of all the three considered RVs; a process that allows for the probabilistically quantified uncertainties to be propagated to the resistance of a given pillar. A histogram was generated from the $10^{4}$ resistance observations for each considered pillar case. The corresponding population parameters are obtained from maximum likelihood estimations from the sample statistics and the resistance was found to follow a normal distribution for all examined cases, as indicatively shown in Figure 5. The corresponding deterministic resistance level obtained from Eq. 8 (as per CSR for the nominal $\mathrm{E}=210 \mathrm{GPa}$ and $\left.\sigma_{\mathrm{y}}=235 \mathrm{MPa}\right)$ and the maximum allowable utilization resistance level $\left(\eta_{\text {all. }}=0.65\right)$ are shown as well.
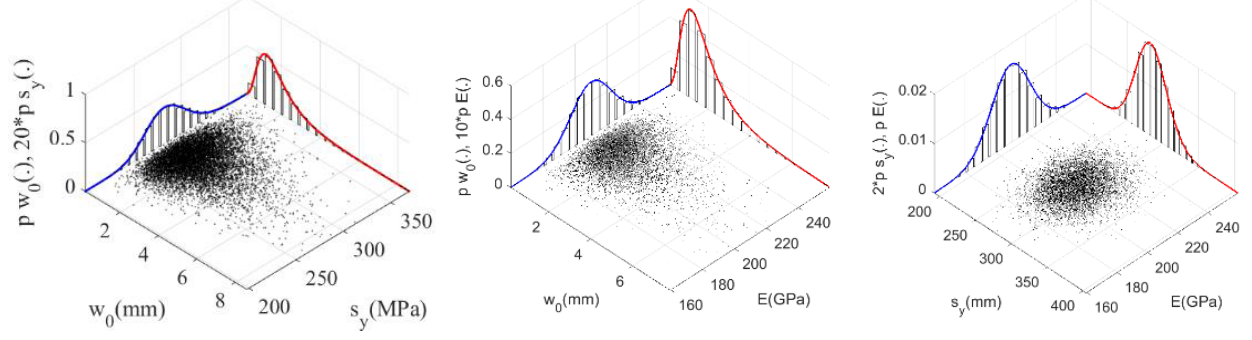

Fig. 4 Latin Hypercube sampling for MCS visualized through pairs of RVs.

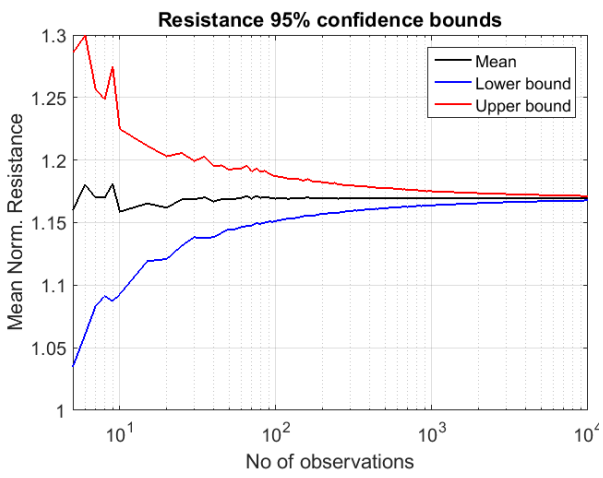

(a)

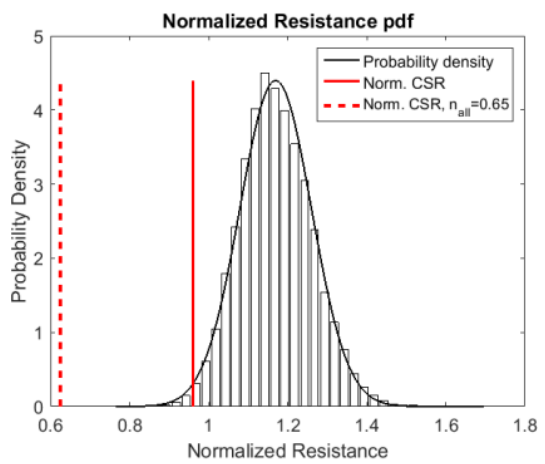

(b)

Fig. 5 MCS convergence plot (a) and corresponding statistical structure (b). 


\section{Parametric MCS and regression}

Three mostly employed cross-section profiles in ship structures were considered for resistance calculation in probabilistic terms, i.e. hollow circular (CHS), hollow square (SHS) and " $\mathrm{H}$ " shape cross-section (HEA). Each profile was probabilistically evaluated at a low, intermediate and high slenderness ratio, $\lambda$, corresponding at pillars with a failure mode mostly characterized by material plasticity, structural instability and material plasticity (elastoplastic buckling) and structural instability, respectively. In order for the parametric analysis to attain statistical significance, ten different pillar scantlings with an equal target slenderness ratio, i.e. $\lambda \approx 0.4,1$ and 1.6 , have been generated per profile. The scantlings were selected such that local buckling was avoided, according to formulas found in [2]. For each pillar case, a MCS has been performed based on the prescription provided in subsection 3.3, resulting in total 30 MCSs per profile ( $3 \lambda$ levels x 10 scantlings).

The unified probability density function (pdf) of the resistance per slenderness ratio was calculated by averaging the sample statistics obtained from all three considered profiles (CHS, SHS and HEA) and the outcome is presented in Figure 5. By considering a one-sided $95 \%$ lower confidence bound for the evaluated pillar cases, one may obtain the respective resistance associated with a given probability of failure $\left(p_{\mathrm{f}}\right)$. For illustration purposes, the one-sided $0.1 \%$ and $0.01 \%$ lower confidence bound resistance levels per slenderness ratio and their respective regressions are presented. Such regressions for the probabilistic resistance may be serve the purpose as buckling resistance curves as an alternative to deterministic based resistance curves, where the notion of a safety factor is employed. The probabilities of failure from the frequentist point of view, denote that from the 95 out of 100 pillar cases with equal $\lambda$, there will be at most 1 out of $1000\left(p_{\mathrm{f}}=0.1 \%\right)$ or 1 out of 10,000 $\left(p_{\mathrm{f}}=0.01 \%\right)$ pillars that will fail if a force smaller than the corresponding resistance will be exerted. It must be noted that the safe design zone as per $\operatorname{CSR}\left(\eta=\sigma / \sigma_{\mathrm{cr}}<\eta_{\text {all. }}\right)$ gives at most a $p_{\mathrm{f}} \approx 1 / 10^{-8}$.

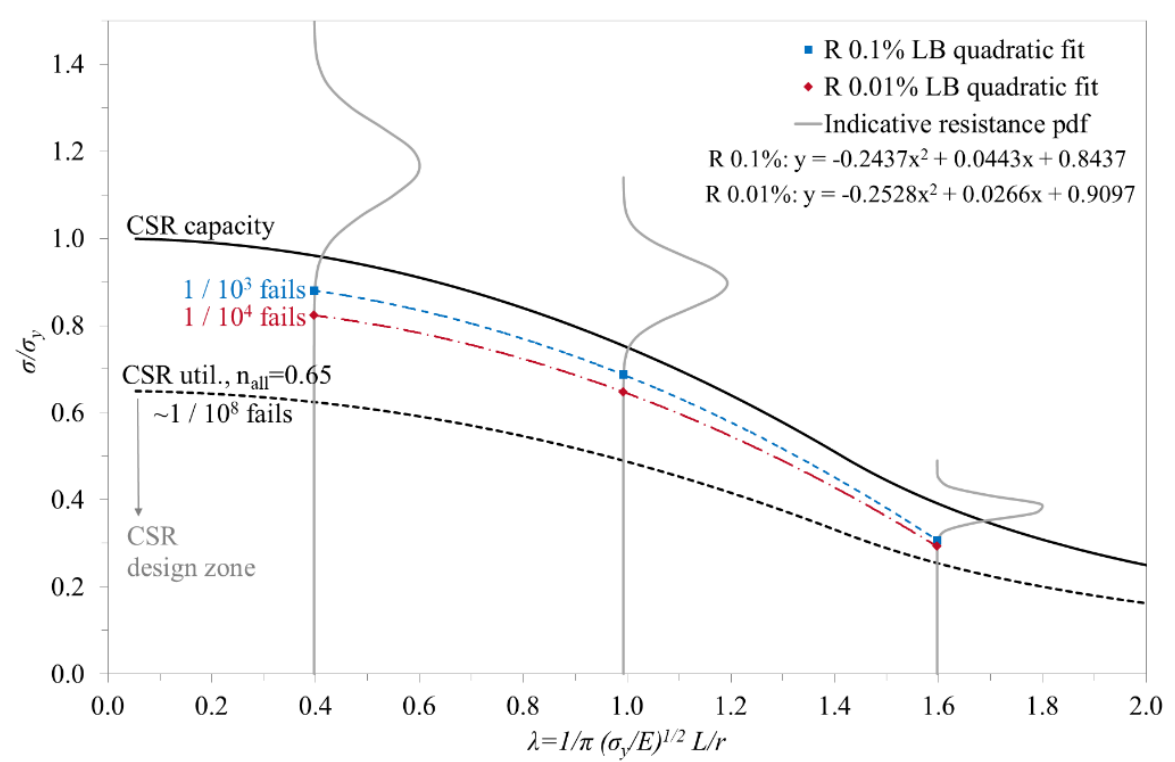

Fig. 6 Deterministic and probabilistic resistance curves (buckling curves).

By controlling the probability of failure, the maximum allowable utilization factor can be selected to accommodate higher risk within the pillar design process (Figure 7). 


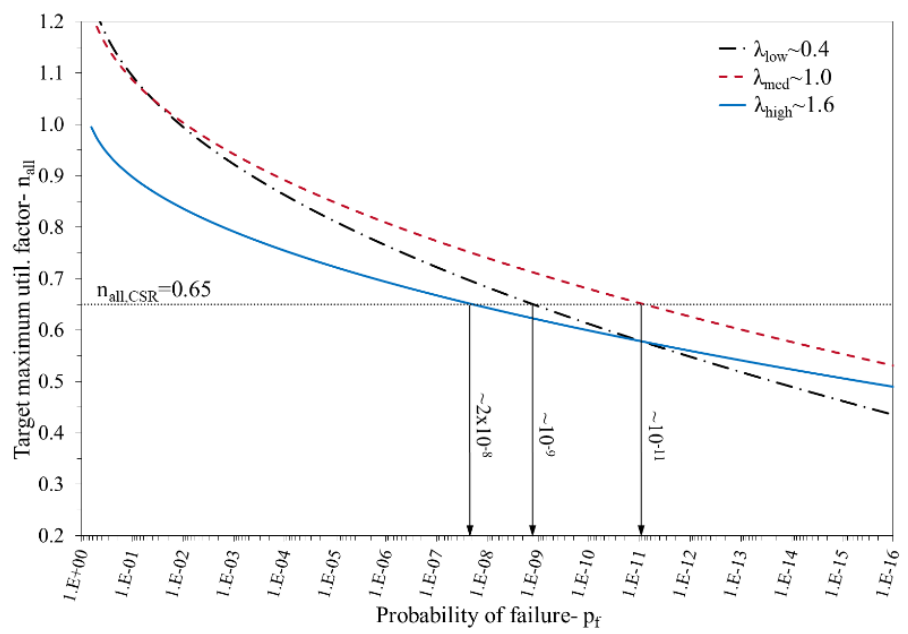

Fig. 7 Transformation of probabilistic to deterministic buckling strength analysis.

\section{Concluding remarks}

Analysis has shown that the probability of failure associated with deterministic rule-base strength prediction with maximum allowable utilization factors is relatively low, i.e. $\sim 1 / 10^{8}$ pillars might fail, meaning that regulations are rather conservative regarding the ultimate pillar compression resistance. By conducting a risk based analysis and by controlling the maximum allowable utilization factor, the failure probability can be selected, so as to accommodate higher risk if required.

\section{References}

1. O.F. Hughes, J.K. Paik, Ship Structural Analysis and Design (The Society of Naval Architects and Marine Engineers, 2010)

2. International Association of Classification Societies (IACS), "Common Structural Rules for Bulk Carriers and Oil Tankers (CSR)" Issued at 1 January 2021

3. R. Ranganathan, Structural Reliability Analysis and Design (Jaico Publishing House, Mumbai, 2006).

4. IACS, "Shipbuilding and Repair Quality Standard," Rec. Rev.8 2017, London, 2017.

5. Z. Kala, J. Kala, M. Škaloud, B. Teplý, "Sensitivity analysis of the effect of initial imperfections on the (i) ultimate load and (ii) fatigue behaviour of steel plate girders" J. of Civil Eng. and Manag. 11, 99 (2005)

6. J. Melcher, Z. Kala, M. Holický, M. Fajkus, L. Rozlívka, " Design characteristics of structural steels based on statistical analysis of metallurgical products" J. of Con. Steel Res. 60, 795 (2003).

7. C. Dou, Y.-L. Pi, "Effects of Geometric Imperfections on Flexural Buckling Resistance of Laterally Braced Columns" J. of Str. Eng. 142, (2017).

8. Z. Kala, "Stability problems of steel structures in the presence of stochastic and fuzzy uncertainty" Thin. Wall. Struct. 45, 861 (2007).

9. Z. Kala, (2011). "Sensitivity analysis of steel plane frames with initial imperfections." Eng. Struct., 33, 2342 (2011). 